EPJ Web of Conferences 21, 10006 (2012)

DOI: $10.1051 /$ epjconf/20122110006

(c) Owned by the authors, published by EDP Sciences, 2012

\title{
Modeling spallation reactions in tungsten and uranium targets with the Geant4 toolkit ${ }^{\star}$
}

\author{
Yury Malyshkin ${ }^{1, a}$, Igor Pshenichnov ${ }^{1,2}$, Igor Mishustin ${ }^{1,3}$, and Walter Greiner ${ }^{1}$ \\ 1 Frankfurt Institute for Advanced Studies, J.-W. Goethe University, 60438 Frankfurt am Main, Ger- \\ many \\ 2 Institute for Nuclear Research, Russian Academy of Science, 117312 Moscow, Russia \\ 3 Kurchatov Institute, Russian Research Center, 123182 Moscow, Russia
}

\begin{abstract}
We study primary and secondary reactions induced by $600 \mathrm{MeV}$ proton beams in monolithic cylindrical targets made of natural tungsten and uranium by using Monte Carlo simulations with the Geant4 toolkit [1-3]. Bertini intranuclear cascade model, Binary cascade model and IntraNuclear Cascade Liège (INCL) with ABLA model [4] were used as calculational options to describe nuclear reactions. Fission cross sections, neutron multiplicity and mass distributions of fragments for ${ }^{238} \mathrm{U}$ fission induced by 25.6 and 62.9 $\mathrm{MeV}$ protons are calculated and compared to recent experimental data [5]. Time distributions of neutron leakage from the targets and heat depositions are calculated.
\end{abstract}

\section{Motivation}

One of the most important applications of high-energy accelerators is to produce neutrons in protoninduced spallation reactions on extended targets made of heavy materials. In this way neutron spallation sources operate, see, e.g., [6] and [7], providing neutrons for neutron scattering experiments and material research. Neutrons produced by energetic protons can be also used to maintain a chain of nuclear fission reactions in a subcritical assembly of an accelerator-driven system (ADS) for nuclear waste incineration [8,9]. In all such cases the neutron flux around the spallation target has to be thoroughly quantified. In particular, the time dependence of the neutron flux on the surface of the spallation target is of a special concern.

\section{Simulations}

Neutron production by $600 \mathrm{MeV}$ proton beam in monolithic non-fissile and fissile cylindrical targets are studied my Monte Carlo Simulations. A dedicated software called MCADS (Monte Carlo for Accelerated Driven Systems) was created in FIAS. It is based on the Geant4 toolkit, which is widely used nowadays in nuclear and particle physics.

We consider spallation targets made of natural tungsten, uranium and also of ${ }^{238} \mathrm{U}$. The latter material was used in validating Geant4 fission models. The radius of these cylindrical targets was taken as $10 \mathrm{~cm}$ and the length as (1) $1 \mathrm{~cm}$ for the fission studies making multiple interactions of beam protons improbable, and (2) $20 \mathrm{~cm}$ for the neutron yields and energy deposition calculations ensuring that all beam protons are absorbed inside the target.

Visualization of a history of one proton and all secondary particles is shown on Fig. 1.

\footnotetext{
^ This project is supported by Siemens Corporate Technology.

a e-mail: malyshkin@fias.uni-frankfurt.de
}

This is an Open Access article distributed under the terms of the Creative Commons Attribution-Noncommercial License 3.0, which permits unrestricted use, distribution, and reproduction in any noncommercial medium, provided the original work is properly cited. 


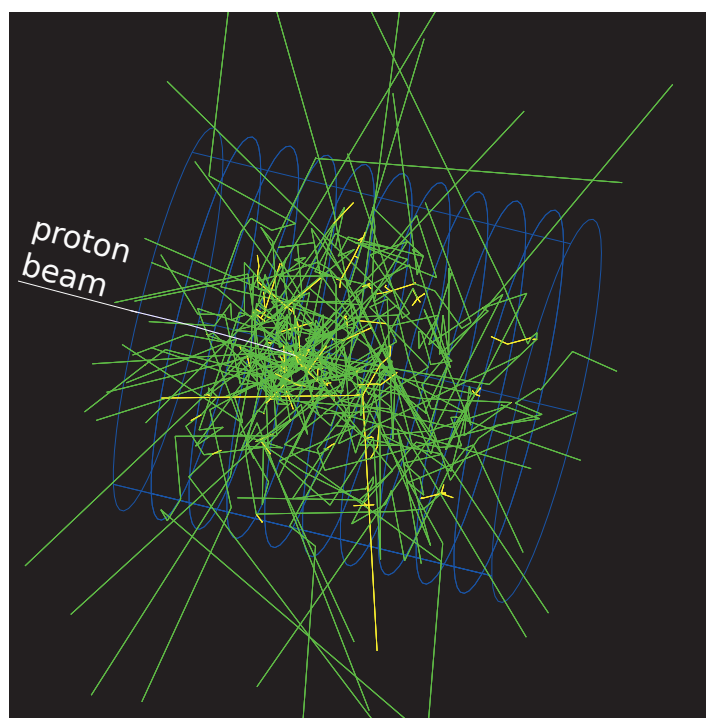

Fig. 1. Visualization of a history of a single proton which hits the uranium target. Green lines - neutron tracks, yellow lines - gamma tracks.

\section{Main physical processes}

Beam protons entering a spallation target lose their energy due to (1) ionization of target atoms with emission of $\delta$-electrons; (2) inelastic nuclear reactions with target atomic nuclei. It is quite common to consider inelastic interactions of fast nucleons with atomic nuclei as consisting of three stages:

- initial fast stage of reaction when a projectile initiates a cascade of collisions with nucleons of the target nucleus;

- preequilibrium process when fast particles leave a highly excited nuclear system;

- deexcitation process of an equilibrated nuclear residue by evaporation of nucleons or by fission.

It is assumed that at the end of the initial stage after the escape of all fast particles the evolution of the remaining nuclear system changes its character. Because of intensive interaction between nucleons, the residual nuclei evolve toward statistical equilibrium. After that at the last stage of the reaction an excited compound nucleus undergoes deexcitation by evaporating nucleons or by fission.

\subsection{Models used in simulations}

The Geant4 toolkit provides several sets of physics models (the so-called Physics Lists [10]) which simulate interactions of protons and neutrons with atomic nuclei. In the considered proton beam energy range below $1 \mathrm{GeV}$ the following Physics Lists are available:

- QGSP_BERT_HP — includes Bertini intranuclear cascade model. The development of Bertini cascade model followed the main ideas of the INUCL Fortran code, which was build as an all-inclusive model, with its own precompound and deexcitation models. An interface to the native Geant 4 preequilibrium and deexcitation is foreseen to be developed in the near future, but currently it is not available.

- QGSP_BIC_HP — includes Binary cascade model. This model simulates a time-dependent development of intranuclear cascades induced by projectile nucleons in target nuclei. The model has been created inside the Geant 4 collaboration, and it is an in house development. It includes native preequilibruim and deexcitation models of the Geant4. 

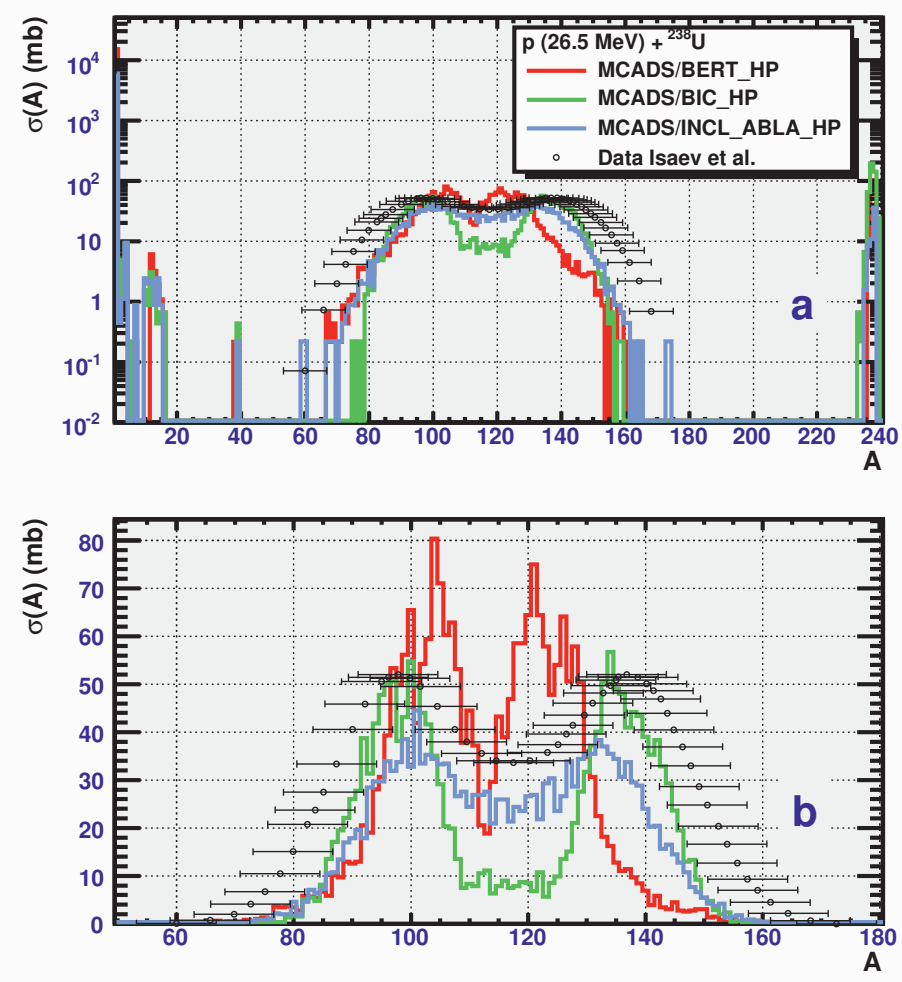

Fig. 2. Mass distribution of nuclear fragments produced in inelastic interactions (a) and only in fission reactions (b) of $26.5 \mathrm{MeV}$ protons with the uranium $\left({ }^{238} \mathrm{U}\right)$ target. Results of simulation using different models and experimental data [5] are presented.

- QGSP_INCL_ABLA - Intra-Nuclear Cascade Liège (INCL) which was developed in Liège. It uses the deexcitation fission-evaporation model for spallation reactions (ABLA), which was developed at GSI, Darmstadt. INCL does not include any preequilibrium model, and therefore may be less accurate for the nucleon-induced reactions below 50-100 MeV.

QGSP_BERT_HP and QGSP_BIC_HP include high-precision methods for transporting neutrons with energies below $20 \mathrm{MeV}$ (NeutronHP). These physics lists were used in MCADS simulations without any modifications. The physics list QGSP_INCL_ABLA was extended by adding NeutronHP methods. The resulting physics list was used in simulations and it is referred in the following as QGSP_INCL_ABLA_HP.

The standard electromagnetic physics package of Geant 4 which accounts for the stochastic nature of the energy loss (energy and range straggling) was used in simulations together with each option of nuclear reaction models.

\section{Fission models validating}

A set of simulation with thin targets (see Sect.2) made of ${ }^{238} \mathrm{U}$ was performed to validate fission reactions modelling by MCADS. Mass distributions of nuclear fragments (see Fig. 2 and Fig. 3), fission cross sections and neutron multiplicity (see Table 1) were obtained.

All three physics lists were tested with the data on proton-induced fission of ${ }^{238} \mathrm{U}$ at 26.5 and $62.9 \mathrm{MeV}$ beam energies. All the considered models underestimate fission cross sections at $26.5 \mathrm{MeV}$. Both Bertini and Binary cascade models predict mass distributions of fission products which differ 

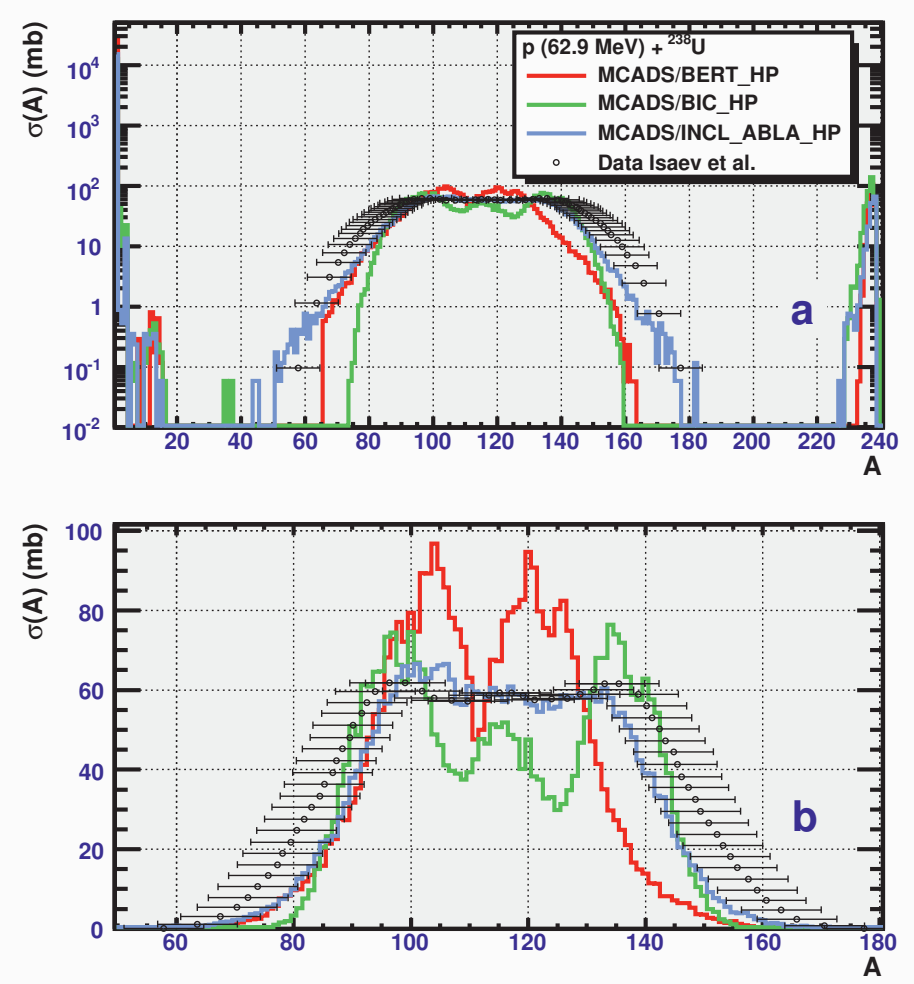

Fig. 3. Same as in Fig. 2, but for fission induced by $62.9 \mathrm{MeV}$ protons.

Table 1. Cross sections and neutron multiplicities in ${ }^{238} \mathrm{U}$ fission induced by $26.5 \mathrm{MeV}$ and $62.9 \mathrm{MeV}$ protons.

\begin{tabular}{lllll}
\hline & \multicolumn{2}{c}{$E_{p}=26.5 \mathrm{MeV}$} & \multicolumn{2}{c}{$E_{p}=62.9 \mathrm{MeV}$} \\
& $\sigma_{f}(\mathrm{mb})$ & $v$ & $\sigma_{f}(\mathrm{mb})$ & $v$ \\
\hline MCADS/BERT_HP & 1101 & 13.44 & 1734 & 14.97 \\
MCADS/BIC_HP & 870 & 5.16 & 1601 & 7.17 \\
MCADS/INCL_ABLA_HP & 876 & 6.13 & 1723 & 8.49 \\
Data Iasev et al. [5] & $1540 \pm 77$ & $4.75 \pm 1.42$ & $1980 \pm 99$ & $6.47 \pm 0.67$ \\
\hline
\end{tabular}

from the measured ones [5]. Moreover, depending on the proton energy Bertini cascade model overestimates the multiplicity of fission neutrons by a factor of $\sim 2$ or 3 .

In general, INCL model gives better results in comparison to other considered models. Fission neutron multiplicity and mass distribution of fission products calculated with this model agree well with the data [5].

\section{Results for extended targets}

Secondary neutrons are produced in the target as products of various reactions, namely, primary spallation reactions, fission, secondary $(n, 2 n),(n, 3 n)$ and $(n, 4 n)$ reactions. Some neutrons are absorbed by radiative capture $(\mathrm{n}, \gamma)$.

Simulations with extended target described in Sect. 2 were performed for non-fissile and fissile targets to investigate the influence of fission ability of a target on neutron production rate. 


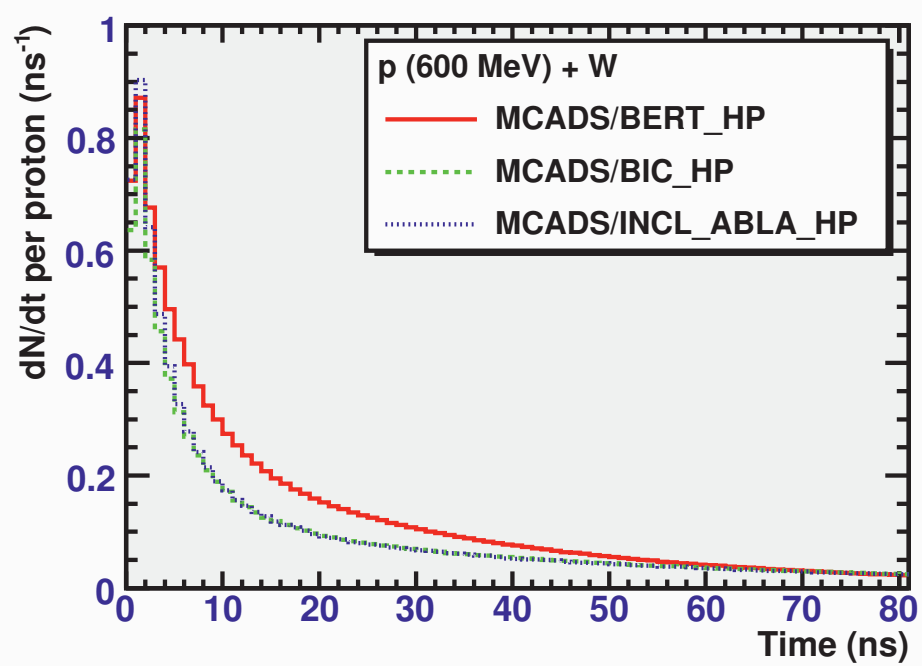

Fig. 4. Time distributions of neutron leakage for non-fissile target made of tungsten.

Table 2. Heat deposition $E_{d e p}(\mathrm{MeV})$ calculated per beam particle. Numbers in parenthesis refer to the calculations neglecting interactions of secondary neutrons.

\begin{tabular}{lcc}
\hline & $\begin{array}{c}\text { Tungsten } \\
\text { target }\end{array}$ & $\begin{array}{c}\text { Uranium } \\
\text { target }\end{array}$ \\
\hline MCADS/BERT_HP & $441(396)$ & $1475(454)$ \\
MCADS/BIC_HP & $452(398)$ & $1189(499)$ \\
MCADS/INCL_ABLA_HP & $445(395)$ & $1383(510)$ \\
\hline
\end{tabular}

\subsection{Time distributions of produced neutrons}

Time distributions of neutron leakage from non-fissile and fissile targets are shown in Fig. 4, 5. The neutron yields calculated with various cascade models mostly agree with each other for the tungsten target but diverge for the uranium one. This is due to the differences in the description of nuclear fission by the considered cascade models. Fission contributes significantly to neutron production: the total number of leaking neutrons is $2.2 \div 3.2$ times higher for the fissile target.

The peak in the leakage time distribution for neutrons is twice as broader for the fissile target (see Fig. 6). This is because of a lower average energy of produced neutrons in the presence of fission reactions.

\subsection{Energy deposition in the target}

The total energy deposition in the target is calculated and presented in Table 2. One can see that absolute value of heat deposition in uranium is generally three times more than in tungsten due to much larger neutron yields.

Histories of transportation of each individual projectile proton in the target are very different due to the stochastic nature of energy deposition by particles in extended media. The distribution of the energy deposited by each single beam proton and divided by the beam energy, $E_{\text {dep }} / E_{\text {beam }}$, is shown in Fig. 7. These results were obtained on event-by-event basis for the tungsten and uranium targets with QGSP_BERT_HP. The distributions exhibit prominent peaks at $E_{\text {dep }} / E_{\text {beam }} \sim 1$ due to the protons, 


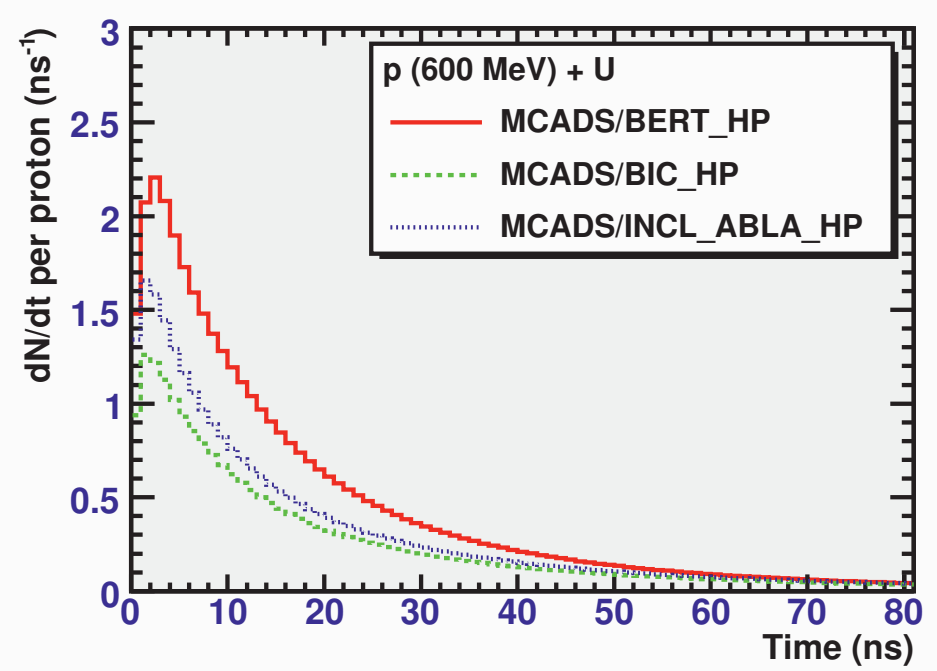

Fig. 5. Time distributions of neutron leakage for fissile target made of uranium.

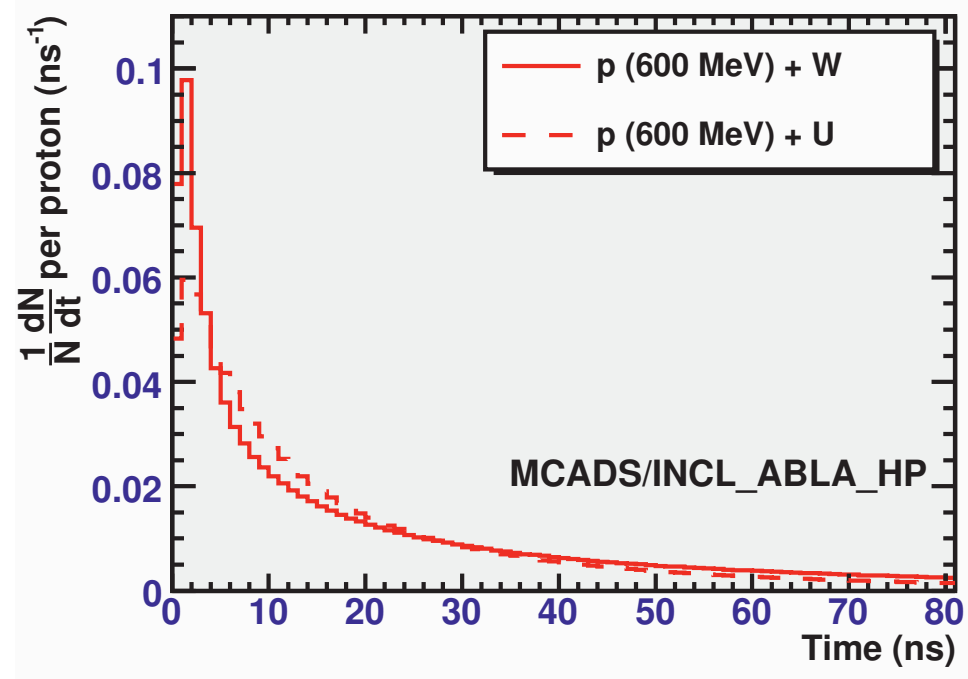

Fig. 6. Same as in Fig. 4 and Fif. 5 but normalized to neutron multiplicity.

which lost their energies only in electromagnetic processes. Such protons do not induce any nuclear reactions neither in tungsten nor in uranium, and their kinetic energy is fully dissipated and converted into target heating which amounts to the initial beam energy. In the case a beam proton initiates a nuclear reaction inside the spallation target, a large part of its energy is converted into kinetic energy of secondary particles, which may leave the target. This is reflected in a reduced energy deposition in such events. The distribution of $E_{d e p} / E_{\text {beam }}$ for such events is very broad with a maximum close to 0.7 for tungsten. Due to the energy released in fission events, the distribution for the uranium target is extended beyond $E_{\text {dep }} / E_{\text {beam }}=1$. 


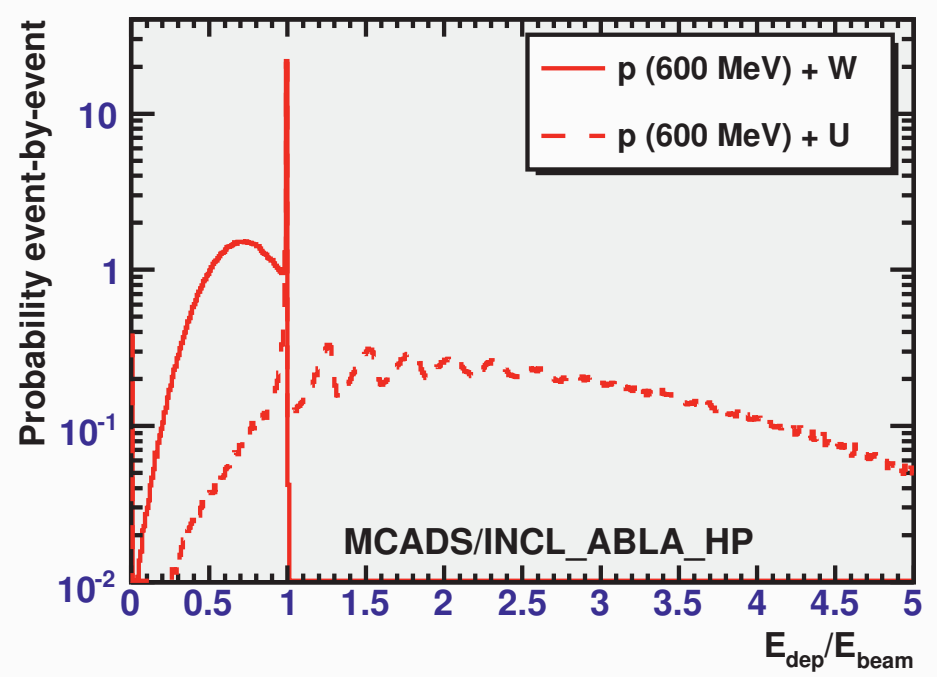

Fig. 7. Probability distribution of the fraction of the beam energy deposited by a single beam proton in tungsten and uranium targets calculated on event-by-event basis.

\section{Conclusions}

Considered cascade models (Bertini, Binary and INCL/ABLA) predict different mass distribution of fission fragments and neutron multiplicity. Comparison with latest experimental data [5] shows that INCL/ABLA gives much better results on proton induced fission of ${ }^{238} \mathrm{U}$ compared to other models.

The role of fissility of the target material is investigated. It was obtained that three times more neutrons are produced by $600 \mathrm{MeV}$ protons on the fissile target, according to the MCADS results using INCL/ABLA model. However, the energy deposition also increases by factor 3 for the uranium target. Optimization studies regarding the neutron flux and energy deposition are in progress.

\section{References}

1. S. Agostinelli et al. (Geant4 Collaboration), Nuclear Instruments and Methods A 506, (2003) 250

2. J. Allison et al. (Geant4 Collaboration), IEEE Transactions on Nuclear Science 53, (2006) 270

3. J. Apostolakis et al. (Geant4 Collaboration), Radiation Physics and Chemistry 78, (2009) 859

4. A. Heikkinen, P. Kaitaniemi and A. Boudard, Journal of Physics Conference Series 119, (2008) 032024

5. S. Isaev et al., Nuclear Physics A, 809, (2008) 1-29.

6. ISIS pulsed neutron and muon source at the Rutherford Appleton Laboratory in Oxfordshire, http://www.isis.stfc.ac.uk/.

7. Spallation Neutron Source (SNS) at Oak Ridge National Laboratory (ORNL), http://myrrha.sckcen.be/.

8. MYRRHA: Multi-purpose hybrid research reactor for high-tech applications, http://myrrha.sckcen.be/.

9. B. Thomauske et al., Aachen Nuclear Safety Reports (ANSR) 1, (2001).

10. Geant4 Physics Reference Manual, http://geant4.web.cern.ch/geant4/support/userdocuments.shtml. 